\title{
Combination therapy for advanced hepatocellular carcinoma: do we see the light at the end of the tunnel?
}

\author{
Ti Zhang ${ }^{1}$, Philippe Merle ${ }^{2}$, Huaqi Wang ${ }^{3}$, Haitao Zhao $^{4}$, Masatoshi Kudo ${ }^{5}$ \\ ${ }^{1}$ Department of Hepatic Surgery, Fudan University Shanghai Cancer Center, Shanghai Medical College, Fudan University, Shanghai, China; \\ ${ }^{2}$ Department of Hepatology, Hôpital de la Croix-Rousse, Hospices Civils de Lyon, Université Lyon 1, 103 Grande rue de la Croix Rousse, Lyon, \\ France; ${ }^{3}$ Department of Hepatobiliary Surgery, Tianjin Medical University Cancer Institute and Hospital, Tianjin, China; ${ }^{4}$ Department of Liver \\ Surgery, Peking Union Medical College Hospital, Chinese Academy of Medical Sciences \& Peking Union Medical College (CAMS \& PUMC), \\ Beijing, China; ${ }^{5}$ Department of Gastroenterology and Hepatology, Kindai University Faculty of Medicine, Osaka-Sayama, Japan \\ Contributions: (I) Conception and design: All authors; (II) Administrative support: None; (III) Provision of study materials or patients: All authors; \\ (IV) Collection and assembly of data: All authors; (V) Data analysis and interpretation: All authors; (VI) Manuscript writing: All authors; (VII) Final \\ approval of manuscript: All authors. \\ Correspondence to: Ti Zhang. Department of Hepatic Surgery, Fudan University Shanghai Cancer Center, Shanghai Medical College, Fudan \\ University, Shanghai 200032, China. Email: zhangti0929@126.com.
}

Importance: Combination therapies of anti-PD-1 and anti-angiogenesis regimens are emerging rapidly and exhibit more promising anti-tumor efficacy for advanced hepatocellular carcinoma (HCC), and consistently it is the hotspot in clinical studies.

Objective: To elaborate several issues which are warranted further consideration as more regimens are being investigated in combination therapies.

Evidence Review: We searched PubMed, MEDLINE, Cochrane Library and Google Scholar by 2021 February for publications on combination therapies for HCC.

Findings: Several clinical issues are worth reconsidering, such as the evaluation on appropriate primary endpoints in phase III clinical trials as for different practical problems, the translation of surrogate endpoint objective response rate (ORR) benefits into overall survival (OS) benefits, and whether conversion surgery contributes to initial expectations of long-term survival or not. New concepts in novel immunotherapy and targeted therapy in combination with loco-regional therapies may improve overall survival for HCC.

Conclusions and Relevance for Reviews: Comprehensive understanding of the mechanism of immunotherapy and targeted therapy contributes to better prognosis of advanced HCC and more explorative combination therapies are needed.

Keywords: Hepatocellular carcinoma (HCC); angiogenesis; immunotherapy; combination therapy

Submitted Dec 01, 2020. Accepted for publication Mar 02, 2021.

doi: 10.21037/hbsn-2021-7

View this article at: http://dx.doi.org/10.21037/hbsn-2021-7

\section{Introduction}

Hepatocellular carcinoma (HCC) is the $4^{\text {th }}$ leading cause of cancer-related death worldwide, with approximately $47 \%$ of new cases in the world occurring in China (1). Specifically, around $70-80 \%$ of patients with HCC are diagnosed at an advanced stage (2), at which time radical treatment is not feasible. Patients with advanced HCC typically receive multimodal therapy primarily comprising systemic treatments. Globally, the 5 -year survival rate associated with HCC is a mere $18.0-19.6 \%(3,4)$ with an exception of $50.4 \%$ in Japan $(5,6)$. In the following review, we summarize the current treatment landscape for advanced HCC, and outline several key challenges for the development of novel combination therapies and treatment strategies. We present the following article in accordance with the Narrative 
Table 1 Ongoing clinical trials investigating the combination of immunotherapy and AADs in advanced hepatocellular carcinoma

\begin{tabular}{lcccc}
\hline Line & Phase & Experimental treatment & Control & Clinicaltrials.gov \\
\hline Second & I & Nivolumab + bevacizumab & - & NCT03382886 \\
First & II & Nivolumab + sorafenib & - & NCT03439891 \\
First & Ib & Nivolumab + lenvatinib & NCT03418922 \\
First & III & Atezolizumab + cabozantinib & Sorafenib & NCT03755791 \\
First & I/II & Tislelizumab + sitravatinib & Sitravatinib & CTR20182363 \\
First & Ib & Pembrolizumab + regorafenib & - & NCT03347292 \\
First & III & Pembrolizumab + lenvatinib & Lenvatinib & NCT03713593 \\
First & Ib & Avelumab + axitinib & - & NCT03289533 \\
Second & I/II & Avelumab + regorafenib & NCT03475953 \\
First & III & Camrelizumab + apatinib & Sorafenib & NCT03764293 \\
\hline
\end{tabular}

Review reporting checklist (available at https://hbsn. amegroups.com/article/view/10.21037/hbsn-2021-7/rc).

\section{Historical landmarks in the development of systemic therapies for HCC}

Until recently, sorafenib (7) and lenvatinib (8) were the only targeted therapies approved for use as first-line treatment for advanced HCC. Despite a continuous search for effective drug treatments for advanced HCC over the past decades, there have been no significant improvements in patient survival. Based on CheckMate 040 (9) and KEYNOTE 224 (10), nivolumab and pembrolizumab were approved as the second-line treatment after sorafenib. However, in 2019, the results of Phase III CheckMate 459 in first line versus sorafenib (11) and Phase III KEYNOTE 240 in second line versus placebo (12) were negative, leading to a failure in approval globally. Actually, neither of these drugs were able to demonstrate the statistically significant survival benefits. In 2020, Finn et al. (13) reported the results of IMbrave 150, a clinical trial in which the combination of atezolizumab [an anti-programmed cell death ligand-1 (PD-L1) monoclonal antibody $(\mathrm{mAb})$ ] and bevacizumab was investigated versus sorafenib for the treatment of advanced HCC in first line setting. The median progression-free survival (mPFS) for this combination therapy was 6.8 months, significantly longer than 4.3 months for sorafenib monotherapy. Although the median overall survival (mOS) was not reached in the combination therapy group, it was significantly longer than the 13.2-month mOS in the sorafenib group (HR $=0.58,95 \% \mathrm{CI}, 0.42-0.79)$. Thus, combined atezolizumab and bevacizumab was the first treatment to show a superior survival outcome to sorafenib. IMbrave 150 was also the first successful phase III clinical trial involving the combination of an immunotherapeutic agent and an anti-angiogenic drug (AAD). AADs have the advantages of rapid onset and potent targeting effects in addition to the effect on alteration of tumor immune microenvironment from immune suppressive to immune permissive properties (14-16), while the advantages of immunotherapy are in the extension and durability of the triggered antitumor immune response $(17,18)$. This has led to the search for further combination therapies that exploit the advantages of combination therapy with AADs and immune checkpoint inhibitors (ICIs).

The Cancer-Immunity Cycle was first proposed in 2013 by Chen DS (19), laying the theoretical foundation for immuno-oncology. Defects in any step of the cancer immunity cycle may modulate the Cycle from its optimal state, eventually leading to cancer development and progression (20). The development and progression of HCC are inseparable from the trilogy of chronic liver disease, liver cirrhosis, and carcinogenesis. Based on the Cancer-Immunity Cycle, restoration of the immune microenvironment of HCC would be expected to transform the immunosuppressive "cold tumor" to a "hot tumor" $(21,22)$, which may be the key to improve the effectiveness of immunotherapy in HCC.

Multiple clinical trials investigating combination immunotherapy and AAD strategies in advanced HCC are ongoing (Table 1). In 2020, Finn et al. reported results from the phase Ib KEYNOTE 524 trial, that evaluated the 
Table 2 Summary of data from clinical trials of targeted therapy and immunotherapy in advanced hepatocellular carcinoma

\begin{tabular}{|c|c|c|c|c|c|c|c|}
\hline Clinical trial & Line & Phase & $\begin{array}{l}\text { Median follow-up } \\
\text { time, months }\end{array}$ & $\begin{array}{c}\text { Number at } \\
\text { risk }^{\dagger}\end{array}$ & $\begin{array}{c}\text { Median TTR, } \\
\text { months }(95 \% \mathrm{Cl})\end{array}$ & $\begin{array}{c}\text { Median DOR, } \\
\text { months }(95 \% \mathrm{Cl})\end{array}$ & Reference \\
\hline REFLECT $(\mathrm{n}=478)$ & First & III & 27.7 & $207-253$ & - & - & (8) \\
\hline CM459 $(n=371)$ & First & III & 30.6 & $165-187$ & $3.3(1.6-19.4)$ & $23.3(3.1-34.5)$ & (11) \\
\hline KN240 (n=278) & Second & III & 13.8 & $135-152$ & $2.7(1.2-16.9)$ & $13.8(1.5-23.6)$ & (12) \\
\hline IMbrave150 $(\mathrm{n}=336)$ & First & III & 15.6 & - & $2.8(1.1-11.3)$ & - & $(13,29)$ \\
\hline
\end{tabular}

TTR, time to response; DOR, duration of response; $\mathrm{Cl}$, confidence interval; NE, not estimable. ${ }^{\dagger}$ Number at risk: the number of survivors corresponding to the median survival time.

efficacy of dual-combination therapy with lenvatinib plus pembrolizumab in patients with unresectable HCC (23). The study reported promising results, showing a mOS of 22 months, mPFS of 9.3 months, median duration of response (mDOR) of 8.6 months, objective response rate (ORR) of $46 \%$ per modified RECIST, and a disease control rate (DCR) of $88 \%$. Real-world data from Taiwan also confirm the excellent therapeutic prospects for lenvatinib plus pembrolizumab in advanced HCC, reporting an ORR and a DCR of $58.7 \%$ and $76.5 \%$ per mRECIST, respectively (24). These data also show lenvatinib plus pembrolizumab has a tolerable safety profile (24). Results from a further phase $\mathrm{Ib}$ clinical trial evaluating lenvatinib plus nivolumab, Study 117, were reported during the 2020 ASCO Gastrointestinal Cancers Symposium. This dualcombination therapy achieved a high ORR of $76.7 \%$ per modified RECIST and a DCR of $96.7 \%$ in patients with unresectable HCC (25). Similarly, a single-arm study by Zhao investigated the combination of pembrolizumab and lenvatinib in advanced biliary tract carcinoma and reported satisfactory outcomes, with an ORR of $25 \%$ and a DCR of $78.1 \%$ per RECIST v1.1 (26). In September 2020, Zhou et al. reported a phase II clinical trial that evaluated the combination of toripalimab [an anti-programmed cell death-1 (PD-1) mAb], lenvatinib, and gemcitabine/ oxaliplatin as first-line treatment for advanced intrahepatic cholangiocarcinoma. The triple-combination therapy achieved excellent outcomes, with an ORR of $80 \%$ and a DCR of $93.3 \%$ (27). It is evident from the results of the above trials that immunotherapy in combination with other targeted therapies exhibit a superior antitumor activity compared to single-agent immunotherapy (28). Nonetheless, current evidence for combination treatment strategies arises from early-stage trials with small sample sizes. The efficacy of combination immunotherapy requires further confirmation in multicenter, prospective, randomized clinical trials. In addition, although the success of the phase III IMbrave 150 has made headway in HCC for the first time in the past 13 years, there are still several background issues warranting further consideration, which we discuss below.

\section{How to evaluate endpoints in phase III clinical trials in HCC?}

\section{Short follow-up duration makes OS ambiguous}

Most current phase III clinical trials of advanced HCC are faced with the challenge of a short median follow-up period that probably affects OS evaluation (Table 2). A short follow-up period will shorten the observation window for target events in clinical trials, which may result in more censored data. The presence of censored data may render it challenging to explain the correlation between the efficacy of anticancer drugs and the OS endpoint typically used in phase III clinical trials. In the initial analysis of data from the IMbrave 150, the median follow-up period for patients in the intent-to-treat population who received atezolizumab plus bevacizumab was 8.9 months. During this follow-up period, the loss to follow-up rate in the combined regimen group was $5 \%$ and in the sorafenib comparator group was $10 \%$. Ninety-six patients died in the combined regimen group $(n=336)$ and 65 died in the sorafenib group $(n=165)(13)$. Therefore, data censoring may have affected the estimation of survival times for the combined regimen and sorafenib. Nevertheless, the combined regimen still showed superior anti-tumor activity compared with sorafenib. The OS 
curves for the combined regimen and sorafenib groups separated in the early follow-up period and the combined regimen had a consistently higher survival rate versus sorafenib. With the extension of the median follow-up period, the OS data for the combined regimen group was mature [mOS of 19.2 months, $95 \%$ confidence interval (CI): 17.0-23.7], and the survival advantage of atezolizumab plus bevacizumab at updated analysis become less important ( $\mathrm{HR}=0.66)$ as compared with the results $(\mathrm{HR}=0.58)$ from the primary analysis at the first interim analysis (followup period=15.6 months) (29). Here, it is worth noting that in prospective clinical trials, the selection of followup duration depends mainly on the aggressiveness of the disease and the prognosis of the patient population. For instance, a 3-year follow-up is sufficient for a clinical trial of advanced pancreatic cancer, whereas the same 3-year follow-up may be too short for cancers with good prognosis, such as thyroid cancer. Therefore, shortening the follow-up period may lead to the overestimation of the efficacy of the interventions in the treated groups in clinical trials (30).

\section{mOS can be easily misinterpreted}

In KEYNOTE 524, the mOS for the pembrolizumab plus lenvatinib group was 22 months [95\% CI: 20.4-not estimable (NE)] (23), which was significantly prolonged by 12 months compared to the sorafenib group in the 2007 SHARP trial. This result provided the hope of survival times exceeding 2 years for patients with advanced HCC. However, it should be noted that the combination of pembrolizumab and lenvatinib may be effective in subset of patients. This is indicated by the relatively wide $95 \%$ confidence interval for mOS. In addition, nine patients were still alive and followed up to 22 months, and the mOS of the combination group was estimated from these nine patients. Judging from the Kaplan-Meier curve trend, it may be speculated that changes in the survival status of this few number of patients would exert a large influence on the estimation of mOS. Assuming that the median follow-up period was longer than 10.6 months, survival status among the small number of at-risk patients would have changed. In such a case, the horizontal line extending from the 50\% cumulative survival point of the Kaplan-Meier curve and the OS curve of the combination group would intersect earlier or later than at 22 months. This suggests that the reestimated mOS would be shorter or longer than 22 months. The inconformity between the clinical significance of mOS and its corresponding number at risk indicates that estimates of long-term efficacy of the combination of pembrolizumab and lenvatinib in KEYNOTE 524 would be very influenced by the survival status of individual patients. With the extension of the median follow-up period, mOS may easily change (Table 2). At present, its excellent antitumor efficacy of pembrolizumab plus lenvatinib are currently being evaluated through the expansion of the sample size of the phase III LEAP 002 clinical trial (Table 1) and its results are eagerly awaited.

In contrast, the median follow-up period in the phase III CheckMate 459 trial was 30.6 months. The mOS of the nivolumab group was 16.4 months, and the corresponding number at risk at this point on the Kaplan-Meier curve was between 165 and 187 (11), which was higher than the corresponding number at risk in KEYNOTE 524. Therefore, the mOS of 16.4 months in CheckMate 459, due to other factors such as short follow-up period, was less affected by censored data, and hence it may be concluded that the data supporting nivolumab for the treatment of advanced HCC is more robust. In addition, this long follow-up data include the effect of subsequent therapy as well. However, mOS itself is not that important since the most important point in Phase III clinical trial is whether or not the trial meets its primary endpoint of OS based on prespecified statistical estimation.

\section{Heterogeneity of the kinetics of response to ICIs}

Clinical observations have identified high heterogeneity in the immune responses mediated by ICIs such as the time to onset of immune response [time to response (TTR)], depth of the response [complete response (CR), partial response (PR), progressive disease $(\mathrm{PD})$, stable disease (SD)], and the duration of the immune response (DOR). Unlike the antitumor mechanism of conventional chemotherapeutic agents, ICIs do not exert a direct cytotoxic effect. ICIs instead exert an antitumor effect by reversing the immunosuppressive state of $T$ cells, activating the immune system and maintaining antitumor immunity $(14,19)$. This immune response takes time to generate, and hence there is often a lag in clinical efficacy (31). For example, clinical studies have reported that around $10 \%$ of patients with metastatic melanoma treated with ipilimumab, a T lymphocyte-associated protein-4 (CTLA-4) antibody, showed atypical immune responses. Following personalized therapy, a subset of tumors initially exhibited PD, followed by tumor regression (PR or CR) or SD; a phenomenon referred to as pseudo-progression (32). Therefore, a 
sufficiently wide clinical observation window is required in clinical trials to evaluate the antitumor activity of ICIs, such as PD-1 and CTLA-4 mAbs. This will avoid the premature termination of the development of a potentially effective therapeutic drug due to pseudo-progression. However, actual disease progression should be identified as early as possible to avoid delay of subsequent treatments. In fact, in HCC pseudo progression has never been reported in all the clinical trials of ICIs (9-13) as well as in a real-world clinical practice. Therefore, in case of HCC, pseudo progression may not exist or can be negligible.

The median TTR in IMbrave 150 was 2.8 months. However, a subgroup of patients experienced disease remission only after receiving the combination therapy for around 11 months (13). Therefore, combination therapy should be continued when disease control continues. Judgement of PD may be performed carefully in order to avoid possibility of pseudo progression. Maybe any PD should be confirmed by another imaging performed 1 to 2 months later or just left to be waited for clinical observation.

\section{OS is affected by subsequent treatments}

In the phase II KEYNOTE 224 trial, the mOS of pembrolizumab as a second-line treatment following failure with sorafenib in advanced HCC was 12.9 months (10), which was better than the 10.6-month mOS reported for regorafenib in the RESOURCE trial (33). However, in the subsequent phase III trial KEYNOTE 240, differences in the co-endpoints (OS and PFS) between pembrolizumab and placebo were not significant per the prespecified statistical plan although clinically meaningful OS benefit was observed (OS HR 0.781, $\mathrm{P}=0.0238$ ). This failure was probably because $47.4 \%$ of the patients in the placebo group had received subsequent-line treatments, including regorafenib and anti-PD-1 mAb, following disease progression (12). Due to the efficacy of the subsequent-line treatments, the mOS of the placebo group was extended to 10.6 months. This is equivalent to the mOS achieved with sorafenib in the SHARP trial. A further sensitivity analysis showed that if the subsequent treatments were censored, the OS benefit of pembrolizumab would be significantly better than that of placebo. A similar phenomenon was observed in CheckMate 459 where $46 \%$ of the patients assigned to sorafenib in this study received subsequent-line systemic treatment, including anti-PD-1 mAbs, following disease progression. In contrast, only $38 \%$ of patients in the nivolumab group had received subsequent-line treatments (11).
This greatly affected the direct comparison of nivolumab and sorafenib.

In summary, as a primary clinical trial endpoint, OS can be affected by multiple complicated factors such as followup duration and effectiveness of subsequent-line treatments. With the increased use of multimodal treatments, the OS for patients with advanced HCC is becoming longer. This makes it more challenging to evaluate therapeutic efficacy using OS as an endpoint. Surrogate endpoints, such as PFS and ORR, have the advantages of smaller sample size requirements and relatively short follow-up period $(34,35)$. However, ultimately, it is generally not possible to translate PFS and ORR benefits to OS benefits $(36,37)$ although PFS and ORR may be surrogate endpoint in the phase II trial in order to speed up the drug development. Clinical trials are designed to solve practical clinical problems. However, if a certain study cannot solve all the problems of patients with advanced HCC, it is more important to identify the key problems to be solved and the indicators suitable for use as study endpoints. Therefore, the classification and stratification of patients with advanced HCC in clinical trials should be refined based on factors such as the presence or absence of vascular invasion, extrahepatic metastasis, liver function compensation, and general conditions. The study objectives should be carefully optimized, and the study endpoints should be selected objectively to best reflect patient survival benefits. For clinical trials involving patients with advanced HCC who may potentially achieve downstaging and conversion to resectable disease, such as those with solitary lesions, large tumors, and grade Vp1-3 portal vein tumor thrombus (PVTT), the more appropriate primary study endpoints may be ORR or PFS. On the contrary, for patients with advanced HCC and an extremely poor prognosis, such as those with multiple lesions, grade Vp4 PVTT, extrahepatic metastasis, liver functional decompensation, and poor general conditions, the key objectives would be to relieve symptoms, prolong survival, and improve quality of life (QoL). Thus, OS and QoL would be the more appropriate study endpoints.

\section{How to translate ORR benefits to OS benefits?}

The 2018 REFLECT trial (8) revealed that lenvatinib outperformed sorafenib in terms of ORR (18.8\% vs. $6.5 \%)$ per RECIST v1.1, but the approval of lenvatinib was merely based on non-inferiority of OS to that of sorafenib (Table 3). It is apparent that, although the disadvantage of sorafenib therapy is a low ORR, to date its OS benefits have not been 
Table 3 Clinical trials comparing sorafenib with other targeted therapies or immunotherapies

\begin{tabular}{llcccc}
\hline Clinical trial & Line & Phase & Regimen & ORR, \% (RECIST v1.1) & mOS, months \\
\hline LIGHT (38) & First & III & Linifanib vs. sorafenib & 13 vs. 6.9 & 9.1 vs. 9.8 \\
BRISK-FL (39) & First & III & Brivanib vs. sorafenib & 12 vs. 9 & 9.5 vs. 9.9 \\
SUN1170 (40) & First & III & Sunitinib vs. sorafenib & 6.6 vs. 6.1 & 7.9 vs. 10.2 \\
REFLECT (8) & First & III & Lenvatinib vs. sorafenib & 18.8 vs. 6.5 & 13.6 vs. 12.3 \\
CM459 (11) & First & III & Nivolumab vs. sorafenib & 15 vs. 7 & 16.4 vs. 14.7 \\
\hline
\end{tabular}

surpassed by other single-agent targeted therapies (38-40). This may be because once a tumor develops resistance against a targeted therapy, it becomes more resilient as it progresses, thereby outweighing the previously achieved ORR benefit (41). Thus, the translation of ORR benefits into OS benefits will be the key to improve the survival of patients with advanced HCC.

In 2019, He et al. reported the results of a randomized, controlled, phase III clinical trial evaluating the efficacy of combining hepatic arterial infusion chemotherapy (HAIC) with sorafenib in advanced HCC (42). This study compared the combination of sorafenib and the HAIC regimen of oxaliplatin, fluorouracil, and leucovorin (FOLFOX) with sorafenib monotherapy. At the clinical follow-up cutoff date, the combination therapy group had a significantly longer PFS than the sorafenib monotherapy group (7.0 vs. 2.6 months), a significant ORR benefit ( $40.8 \%$ vs. $2.5 \%$ ) and a DCR of $76 \%$ per RECIST, which was much higher than that achieved by existing single-agent targeted therapies and immunotherapies. Most importantly, the mOS for the combination therapy group was also significantly longer than that of the sorafenib monotherapy group (13.37 vs. 7.13 months). Specifically, 16 patients $(12.8 \%)$ in the combination therapy group underwent surgical resection, and three underwent local ablation. Therefore, given the short-term ORR and PFS benefits for HAIC plus sorafenib, an effective way to improve OS with this combination is to proactively conduct radical treatments, such as conversion surgery or ablation. This may also be the key to overcome the challenges of translating ORR and PFS benefits to OS benefits.

In July 2020, Mazzaferro et al. (43) reported the efficacy of liver transplantation following successful downstaging and conversion of advanced HCC beyond the Milan criteria by sequential therapies. In that phase IIb/III trial, 45 patients with advanced HCC beyond the Milan criteria and without macrovascular invasion and extrahepatic metastasis were randomly assigned to a liver transplantation group and non-transplantation group. The tumors in both groups underwent successful downstaging and conversion following local or systemic therapies. Liver transplantation was performed in the experimental group, while in the control group local or systemic treatments were administered only at the time of disease progression following successful downstaging. With a median follow-up of 71 months at the data cutoff, the 5-year tumor-free survival rate and the 5 -year OS rate of the liver transplantation group were significantly higher than those of the control group ( $76.8 \%$ vs. $18.3 \%$ and $77.5 \%$ vs. $31.2 \%$, respectively). The Mazzaferro study is the first prospective, randomized, controlled clinical trial to investigate liver transplantation following downstaging therapies in advanced HCC. The OS benefit in the liver transplantation group suggested that subsequent radical treatment (e.g., transplantation, resection, or ablation) following the successful conversion of advanced HCC significantly prolonged survival.

\section{Combination therapy: dual- or triple-combination therapy? And timing?}

Based on the typical shape of Kaplan-Meier curves for combination immunotherapies, it appears that patients with an effective immune response attain a durable antitumor response at a certain time point (i.e., the onset of the immune response). This is referred to as the 'plateau of long-term survival' phenomenon (44). Existing clinical trials on advanced HCC have reported different TTRs (Table 2). It has been speculated that the addition of local interventional therapy (45) to combined antitumor therapy with anti-vascular endothelial growth factor (VEGF) antibodies and ICIs may be an effective means to shorten TTR and increase ORR in patients with HCC (46). Local chemotherapy delivered through the hepatic artery can destroy HCC tissues directly and lead to apoptosis of 
cancer cells. The apoptotic cancer cells release tumor antigens (47), which improve priming the $\mathrm{T}$ cell and antiVEGF inhibitor further improve the Cancer-Immunity Cycle in the immune microenvironment of HCC, thereby enhancing the capacity of the immune system to destroy cancer cells. A small sample size retrospective study showed that the DCR obtained with HAIC + targeted therapy + anti-PD-1 mAb regimens was $100 \%$, including a CR of $50 \%$ and a PR of $50 \%$ (48), which was significantly higher than the double-combination regimens used in trials such as KEYNOTE 524. The phase III clinical trial, LEAP012 (49), investigating the combination of pembrolizumab, lenvatinib, and transarterial chemoembolization as the treatment for advanced HCC is currently ongoing.

The safety reported in IMbrave 150 does not limit the real-world application of the combination regimen investigated in that study. In IMbrave 150 trial, 25.2\% of the patients experienced a bleeding event of all cause (6.4\% in Grade 3 or 4 ), although there were six events of fatal bleeding or ulcer perforation (13) in combination arm. Grade 5 total event in sorafenib arm is $5.8 \%$ similar to combination arm (4.6\%). Notably, the IMbrave 150 trial included patients with advanced HCC with wellcompensated liver function, such as those with Child-Pugh Class A and without esophageal or gastric varices with low bleeding risk and patients with an intermediate or high risk for portal hypertension were excluded (13). With these stringent enrollment criteria, treatment-related bleeding events were very few. However, application of this drug combination in the real-world setting should be approached with caution similar to the clinical trial.

Further combination therapies may be seeked for patients with HCC who are Child-Pugh class B, with a high risk of bleeding, or PVTT/IVCTT. Compared with IMbrave 150, the KEYNOTE 524 and Study117 trials $(23,25)$ had more stringent requirements regarding liver function, in which only Child-Pugh class A was allowed. Meanwhile, the bleeding risk associated with the combination of antiPD-1 mAb and lenvatinib was also low, while the ORR was relatively high, suggesting this combination may be better regimen for the conversion of advanced HCC prior to surgical resection.

Regardless of the particular dual- or triple-combination regimen comprising HAIC, immunotherapy, or targeted therapy, these combinations are all subject to the risk of additive liver function damage. For example, in the combination group of IMbrave 150, the incidence of grade 3-4 alanine aminotransferase and aspartate aminotransferase increase were $3.6 \%$ and $7 \%$, respectively (13). Meanwhile, the incidence of grade 3 transaminase increase was $11 \%$ in the combination group in KEYNOTE 524 (23). As mentioned previously, ICIs have a long onset of action; the median onset of action of anti-PD-1 mAbs is approximately three months $(10,11)$. Therefore, it is believed that for very advanced BCLC stage C HCC with main portal vein invasion in which the mOS is only about three months $(50,51)$, multimodal triple-combination therapy may be introduced as early as possible by adding ICIs to the HAIC and AADs combination to achieve rapid tumor control. However, some researchers believe that the triple combination regimen of HAIC, ICIs, and AADs exerts additive adverse reactions. Hence, attention should be paid to the incidence of adverse events, such as liver function damage, when using any combination therapy. Consequently, treatment aspects of advanced HCC that have not been clinically investigated, such as the sequence and timing for combining local treatment, AADs, immunotherapy, or other treatments, still merits further exploration. Before implementing these triple regimens in the practice, prospective randomized controlled study is mandatory.

\section{Radiological CR, what to do next?}

Although sorafenib has been the standard treatment regimen for advanced HCC since 2007, its associated ORR of $2 \%(8)$ is suboptimal. In comparison, lenvatinib is associated with a higher ORR (7), and the CR rate (mRECIST criteria) associated with immunotherapy is approximately $11 \%(23,25)$. Also, updated results of IMbrave 150 trial showed atezolizumab plus bevacizumab combination therapy achieved CR in $12 \%$ per mRECIST (29). This implies that a subgroup of patients with advanced HCC that was incurable in the past can now achieve a CR with immunotherapy, and the advantage of immunotherapy is the possibility of achieving a long-term survival once there is an initial response. Whether the next step of treatment following radiological CR should be radical therapy (surgery, transplantation, ablation), maintenance therapy with pharmacologic combinations or observation remains controversial.

In the aforementioned clinical trial led by Mazzaferro (43), the liver transplantation group had a survival benefit of 14.5 months, compared with the control group. A further survival analysis found that the mOS of the subgroup that achieved a PR after downstaging and conversion was 26.5 months, while the mOS in the CR subgroup without 
conversion to liver transplantation was 9.9 months. The authors suggested that the disparity between the two subgroups could be because radiological CR might not represent pathological CR. The result may also be due to a subgroup of patients with radiological CR experiencing disease progression after refusing liver transplantation. In KEYNOTE 524, two patients with radiological CR eventually experienced PD and died, and seven patients with radiological PR experienced PD and died (23). Similarly, in Study 117, with an extended clinical observation period, a subset of advanced HCC that had initially reduced in size grew rapidly $(25,52)$. Kudo et al. proposed a novel treatment strategy for Barcelona Clinic Liver Cancer (BCLC) stage $\mathrm{B}$ and C HCC by suggesting that conversion and surgical resection could be indicated for patients with these stages of disease (53). Reports have shown that the range of the 5-year survival rate for patients with advanced HCC after conversion therapies and secondary surgical resection or liver transplantation is $25-57 \%(54,55)$, which is equivalent to a 5 -year survival rate of $30-60 \%$ in patients with resectable HCC (56). Because of this, it is speculated that patients who have achieved radiological CR following combination immunotherapy should receive curative treatments, such as conversion surgery or local ablation, to achieve longer survival. However, there are currently no prognostic data on the use of combination immunotherapy for downstaging and conversion to surgery, and the effectiveness of this approach needs to be elucidated in future studies.

\section{Dynamic monitoring of PD-1/PD-L1 expression or TIME through tumor biopsy}

Presently, no definitive biomarkers that can predict response to anti-PD-1 mAbs in HCC have been identified. In CheckMate 459, even the patient group with negative PD-1 expression showed an immune response after receiving ICIs, but the response rate was lower than that in the PD-1positive group. However, the ORR of PD-1-positive patients treated with nivolumab was twice that of the PD-1-negative group (28\% vs. $12 \%)$ (11). In the Phase 1b GO30140 study, the cutoff values for positive PD-L1 expression in tumor-infiltrating immune cells or tumor cells were set at $1 \%, 5 \%$, and $10 \%$. With increasing PD-L1 expression cutoffs, the ORR showed an upward trend of $41 \%, 46 \%$, and $50 \%$, respectively (57), however, small patient numbers in some PD-L1 subgroups make interpretation difficult regarding the association between PD-L1 expression and efficacy. This observation may suggest that the AAD, as part of the combination regimen, may alter the PD-1/ PD-L1 expression levels of the abovementioned cells, which increases the response rate achieved with antiPD-L1 mAbs. A preliminary preclinical study has shown that the combination of anti-VEGF and anti-PD-1 mAbs can restore the immune microenvironment, in which the expression of PD-L1 in tumor cells and stromal cells was upregulated, thereby increasing the sensitivity of tumor tissues to anti-PD-1 mAbs (58,59). In IMbrave 150, 64\% $(79 / 124)$ of the patients in the combination therapy group were $\mathrm{PD}$-L1-positive $\geq 1 \%$ in tumor-infiltrating immune cells or tumor cells (13). However, it remained unclear whether the antitumor activity was attributable to the additive or synergistic effect of dual-combination therapy. Due to the lack of repeated biopsies of the tumor tissue, the potential antitumor mechanism of the combination could not be fully interpreted. Therefore, dynamic monitoring of the changes in PD-1/PD-L1 expression in tumor tissue through tumor biopsy before, during, and after treatment may facilitate the delineation of the antitumor mechanism underlying the dual-combination therapy. TIME (tumor immune microenvironment) also plays an important role of the combination therapy. Therefore, dynamic monitoring of Treg or MDSC may also be helpful for the understanding its mechanism (15).

\section{Treatment options following PD-1 resistance}

In IMbrave 150, a subgroup of patients in the combination therapy group received anti-PD-1 mAbs as subsequent treatments (13), but its efficacy is unknown. As mentioned earlier, the TTR of the combination of pembrolizumab or nivolumab with lenvatinib is comparable (1.9 vs. 1.87 months), but the latter is associated with a higher ORR (46\% vs. $76.7 \%$ ) although background characteristics, numbers of patients and observation period are different between those groups $(23,25)$.

When factors such as drug availability and cost are considered (Table 4), orally administered targeted therapies remain the first choice for treating advanced HCC in many economically underdeveloped regions. Compared with the requirements for administering the combination regimens in IMbrave 150, KEYNOTE 524, and Study 117, treatment with HAIC can be completed in an interventional suite with equipment such as digital subtraction angiography. Oxaliplatin, fluorouracil, and leucovorin calcium are also relatively common and inexpensive antitumor drugs. In 
Table 4 Medical costs of clinical trials in advanced hepatocellular carcinoma

\begin{tabular}{|c|c|c|c|c|c|}
\hline Regimen & Route of administration & Administration dosage ${ }^{\ddagger}$ & $\begin{array}{l}\text { Medical-costs } \\
\$ / \text { month }\end{array}$ & Supplement & Reference \\
\hline Sorafenib monotherapy & Oral medication & $400 \mathrm{mg}$ bid & 1,600 & FDA approved & $(7)$ \\
\hline Atezolizumab + bevacizumab & Intravenous injection & $1,200 \mathrm{mg} q 3 w+900 \mathrm{mg} q 3 w$ & $6,400+2,600$ & FDA approved & (13) \\
\hline Pembrolizumab + lenvatinib & $\begin{array}{l}\text { Intravenous injection + oral } \\
\text { medication }\end{array}$ & $12,000 \mathrm{mg} q 3 w+8 \mathrm{mg} q d$ & $7,000+5,000$ & $\begin{array}{l}\text { No indication } \\
\text { in China }\end{array}$ & (23) \\
\hline $\mathrm{HAIC}+\mathrm{PD}-1+\mathrm{AAD}$ & $\begin{array}{l}\text { Transarterial }+ \text { intravenous } \\
\text { infusion }+ \text { oral medication }\end{array}$ & $\begin{array}{l}\text { HAIC q3w + Sintilimab 12,000 mg } \\
\text { q3w + Apatinib } 500 \text { mg qd }\end{array}$ & $\begin{array}{l}2,900 / \text { cycle }+ \\
1,158+1,111\end{array}$ & $\begin{array}{l}\text { No indication } \\
\text { in China }\end{array}$ & \\
\hline
\end{tabular}

${ }^{\ddagger}$ Estimated based on a 60-kg patient. AAD, anti-angiogenic drug. HAIC, hepatic arterial infusion chemotherapy.

China, combination regimens that primarily comprise HAIC, AAD, and domestically manufactured anti-PD-1 $\mathrm{mAb}$ exhibit an excellent cost-effectiveness ratio and accessibility for patients with advanced HCC. However, these data remain to be confirmed in a large prospective validation cohort.

After failure of PD-1/PD-L1 antibody therapy, Aoki et al. (60) reported that lenvatinib monotherapy showed promising outcome (ORR of $55.6 \%$, DCR of $86.1 \%$ per mRECIST, PFS of 10.03 months, mOS of 15.8 months since the initiation of lenvatinib and mOS of 29.8 months since 1st line PD-1/PD-L1 antibody therapy). This favorable outcomes can be explained by prolonged binding effect of ICI antibody to lymphocyte more than 20 weeks after termination of ICIs (61) in addition to immune modulating effect of lenvatinib. In other words, therapeutic effect can be expected similar to lenvatinib and PD-1 antibody combination therapy when lenvatinib is introduced after failure of ICIs.

In addition, PD-1/PD-L1 antibodies do not respond to WNT/ $\beta$-catenin mutated HCC $(62,63)$, however lenvatinib is highly effective for $\mathrm{WNT} / \beta$-catenin mutated HCC since FGFR4 is highly expressed in $\mathrm{WNT} / \beta$-catenin mutated HCC (64).

\section{Conclusions}

A definitive driver gene that can explain the molecular mechanism of HCC has not been identified. Drugs such as sorafenib are merely multitargeted AADs, and not targeted therapies for specific driver genes in HCC. Most treatment strategies for advanced HCC focus on intervention in the immune microenvironment, and the restoration of the immune microenvironment of HCC may be an effective means to improve the efficacy of immunotherapies in advanced HCC. Evidence shows that treatments such as local radiotherapy and oncolytic viruses facilitate the induction of apoptosis in tumor tissues and the release of immunogenic tumor antigens $(47,65)$. The released tumor antigens, in turn, induce changes in the immune microenvironment, favoring the further amplification of the antitumor activity of ICIs. The combination of HAIC-based local therapy with targeted therapy and immunotherapy may be a key direction for multimodal therapies for advanced HCC especially in China. Data from early phase, small-sample studies, as well as unpublished data from our group, show that the triple-combination FOLFOX regimen that comprises HAIC + anti-PD-1 mAbs + AAD can achieve an ORR above $90 \%$, which is better than that achieved by single-agent anti-PD-1 mAbs, AADs, and dual-combination regimens. Efficacy of this triple regimen must be confirmed by prospective RCT. Therefore, the superior antitumor efficacy exhibited by triple-combination regimens may delay the urgent need for solving certain challenges in HCC, such as accurate molecular typing and the search for driver genes. Meanwhile, the search for effective combination regimens might be the key to improve survival in patients with advanced HCC in the near future.

However, outside China combination immunotherapies such as PD-1/PD-L1 antibody plus anti-VEGF/AAD and PD-1/PD-L1 plus anti-CTLA4 antibody are still main stream treatment for advanced HCCs.

\section{Acknowledgments}

Funding: This study was funded by the National Science 
and Technology Major Project (No.2017ZX10203207); the National Natural Science Foundation of China (No. 81672884). The National Science and Technology Major Project and the National Natural Science Foundation of China had no role in the design or conduct of the study; collection, management, analysis, and interpretation of the data; preparation, review, or approval of the manuscript; or the decision to submit for publication.

\section{Footnote}

Reporting Checklist: The authors have completed the Narrative Review reporting checklist. Available at https:// hbsn.amegroups.com/article/view/10.21037/hbsn-2021-7/rc

Conflicts of Interest: All authors have completed the ICMJE uniform disclosure form (available at https://hbsn.amegroups. com/article/view/10.21037/hbsn-2021-7/coif). Dr. TZ reports grants from National Science and Technology Major Project (No. 2017ZX10203207) and National Natural Science Foundation of China (No. 81672884). Dr. PM serves as a member of AdBoard from Roche, AstraZeneca, BMS, MSD, Eisai, Ipsen, Lilly, Bayer, and Research Grant from Ipsen. Dr. HZ serves as unpaid editorial board members of Hepatobiliary Surgery and Nutrition. Dr. MK reports Lecture fee/Honoraria from Eisai, Bayer, MSD, BMS, EA Pharma, Eli Lilly, Chugai; grant from Eisai, Takeda, Otsuka, Taiho, EA Pharma, Gilead Sciences, Abbvie, Sumitomo Dainippon Pharma, Chugai, Ono Pharmaceutical Co.; Grant/ Contracts: research from Ono Pharmaceutical Co.) and Advisory Consulting fee from Eisai, Ono Pharmaceutical Co., MSD, BMS, Roche. The other author has no conflicts of interest to declare.

Ethical Statement: The authors are accountable for all aspects of the work in ensuring that questions related to the accuracy or integrity of any part of the work are appropriately investigated and resolved.

Open Access Statement: This is an Open Access article distributed in accordance with the Creative Commons Attribution-NonCommercial-NoDerivs 4.0 International License (CC BY-NC-ND 4.0), which permits the noncommercial replication and distribution of the article with the strict proviso that no changes or edits are made and the original work is properly cited (including links to both the formal publication through the relevant DOI and the license). See: https://creativecommons.org/licenses/by-nc-nd/4.0/.

\section{References}

1. Bray F, Ferlay J, Soerjomataram I, et al. Global cancer statistics 2018: GLOBOCAN estimates of incidence and mortality worldwide for 36 cancers in 185 countries. CA Cancer J Clin 2018;68:394-424.

2. Park JW, Chen M, Colombo M, et al. Global patterns of hepatocellular carcinoma management from diagnosis to death: the BRIDGE Study. Liver Int 2015;35:2155-66.

3. Jemal A, Ward EM, Johnson CJ, et al. Annual Report to the Nation on the Status of Cancer, 1975-2014, Featuring Survival. J Natl Cancer Inst 2017;109:djx030.

4. Zhang B, Zhang B, Zhang Z, et al. 42,573 cases of hepatectomy in China: a multicenter retrospective investigation. Sci China Life Sci 2018;61:660-70.

5. Kudo M, Izumi N, Kubo S, et al. Report of the 20th Nationwide follow-up survey of primary liver cancer in Japan. Hepatol Res 2020;50:15-46.

6. Kudo M. Management of Hepatocellular Carcinoma in Japan: Current Trends. Liver Cancer 2020;9:1-5.

7. Llovet JM, Ricci S, Mazzaferro V, et al. Sorafenib in advanced hepatocellular carcinoma. $\mathrm{N}$ Engl J Med 2008;359:378-90.

8. Kudo M, Finn RS, Qin S, et al. Lenvatinib versus sorafenib in first-line treatment of patients with unresectable hepatocellular carcinoma: a randomised phase 3 noninferiority trial. Lancet 2018;391:1163-73.

9. El-Khoueiry AB, Sangro B, Yau T, et al. Nivolumab in patients with advanced hepatocellular carcinoma (CheckMate 040): an open-label, non-comparative, phase $1 / 2$ dose escalation and expansion trial. Lancet 2017;389:2492-502.

10. Zhu AX, Finn RS, Edeline J, et al. Pembrolizumab in patients with advanced hepatocellular carcinoma previously treated with sorafenib (KEYNOTE-224): a non-randomised, open-label phase 2 trial. Lancet Oncol 2018;19:940-52.

11. Yau T, Park JW, Finn RS, et al. CheckMate 459: A randomized, multi-center phase III study of nivolumab (NIVO) vs sorafenib (SOR) as first-line (1L) treatment in patients (pts) with advanced hepatocellular carcinoma (aHCC). Ann Oncol 2019;30:v874-5.

12. Finn RS, Ryoo BY, Merle P, et al. Pembrolizumab As Second-Line Therapy in Patients With Advanced Hepatocellular Carcinoma in KEYNOTE-240: A Randomized, Double-Blind, Phase III Trial. J Clin Oncol 2020;38:193-202.

13. Finn RS, Qin S, Ikeda M, et al. Atezolizumab plus 
Bevacizumab in Unresectable Hepatocellular Carcinoma. N Engl J Med 2020;382:1894-905.

14. Kudo M. Scientific Rationale for Combined Immunotherapy with PD-1/PD-L1 Antibodies and VEGF Inhibitors in Advanced Hepatocellular Carcinoma. Cancers (Basel) 2020;12:1089.

15. Kudo M. A New Era in Systemic Therapy for Hepatocellular Carcinoma: Atezolizumab plus Bevacizumab Combination Therapy. Liver Cancer 2020;9:119-37.

16. Kudo M. Immuno-Oncology Therapy for Hepatocellular Carcinoma: Current Status and Ongoing Trials. Liver Cancer 2019;8:221-38.

17. Hales RK, Banchereau J, Ribas A, et al. Assessing oncologic benefit in clinical trials of immunotherapy agents. Ann Oncol 2010;21:1944-51.

18. Sharma P, Allison JP. The future of immune checkpoint therapy. Science 2015;348:56-61.

19. Chen DS, Mellman I. Oncology meets immunology: the cancer-immunity cycle. Immunity 2013;39:1-10.

20. Motz GT, Coukos G. Deciphering and reversing tumor immune suppression. Immunity 2013;39:61-73.

21. Fukumura D, Kloepper J, Amoozgar Z, et al. Enhancing cancer immunotherapy using antiangiogenics: opportunities and challenges. Nat Rev Clin Oncol 2018;15:325-40.

22. Khan KA, Kerbel RS. Improving immunotherapy outcomes with anti-angiogenic treatments and vice versa. Nat Rev Clin Oncol 2018;15:310-24.

23. Finn RS, Ikeda M, Zhu AX, et al. Phase Ib Study of Lenvatinib Plus Pembrolizumab in Patients With Unresectable Hepatocellular Carcinoma. J Clin Oncol 2020;38:2960-70.

24. Wu C-J, editor. Real-world experience of pembrolizumab plus lenvatinib in unresectable hepatocellular carcinoma in Taiwan2020; ASCO Virtual Scientific Program: American Society of Clinical Oncology.

25. Kudo M, editor. A phase Ib study of lenvatinib (LEN) plus nivolumab (NIV) in patients (pts) with unresectable hepatocellular carcinoma (uHCC): Study 1172020; Gastrointestinal Cancers Symposium: American Society of Clinical Oncology.

26. Lin J, Yang X, Long J, et al. Pembrolizumab combined with lenvatinib as non-first-line therapy in patients with refractory biliary tract carcinoma. Hepatobiliary Surg Nutr 2020;9:414-24.

27. Zhou J, Fan J, Shi G, et al. 56P Anti-PD1 antibody toripalimab, lenvatinib and gemox chemotherapy as first- line treatment of advanced and unresectable intrahepatic cholangiocarcinoma: A phase II clinical trial. Ann Oncol 2020;31:S262-3.

28. Kudo M. Limited Impact of Anti-PD-1/PD-L1 Monotherapy for Hepatocellular Carcinoma. Liver Cancer 2020;9:629-39.

29. Finn RS, editor. IMbrave150: Updated overall survival (OS) data from a global, randomized, open-label phase III study of atezolizumab (atezo) + bevacizumab (bev) versus sorafenib (sor) in patients (pts) with unresectable hepatocellular carcinoma (HCC)2021; Gastrointestinal Cancers Symposium: American Society of Clinical Oncology.

30. Clark TG, Bradburn MJ, Love SB, et al. Survival analysis part I: basic concepts and first analyses. Br J Cancer 2003;89:232-8.

31. Queirolo P, Spagnolo F. Atypical responses in patients with advanced melanoma, lung cancer, renal-cell carcinoma and other solid tumors treated with antiPD-1 drugs: A systematic review. Cancer Treat Rev 2017;59:71-8.

32. Saenger YM, Wolchok JD. The heterogeneity of the kinetics of response to ipilimumab in metastatic melanoma: patient cases. Cancer Immun 2008;8:1.

33. Bruix J, Qin S, Merle P, et al. Regorafenib for patients with hepatocellular carcinoma who progressed on sorafenib treatment (RESORCE): a randomised, double-blind, placebo-controlled, phase 3 trial. Lancet 2017;389:56-66.

34. Lencioni R, Montal R, Torres F, et al. Objective response by mRECIST as a predictor and potential surrogate endpoint of overall survival in advanced HCC. J Hepatol 2017;66:1166-72.

35. Llovet JM, Villanueva A, Marrero JA, et al. Trial Design and Endpoints in Hepatocellular Carcinoma: AASLD Consensus Conference. Hepatology 2021;73 Suppl 1:158-91.

36. Tang PA, Bentzen SM, Chen EX, et al. Surrogate end points for median overall survival in metastatic colorectal cancer: literature-based analysis from 39 randomized controlled trials of first-line chemotherapy. J Clin Oncol 2007;25:4562-8.

37. McKee AE, Farrell AT, Pazdur R, et al. The role of the U.S. Food and Drug Administration review process: clinical trial endpoints in oncology. Oncologist 2010;15 Suppl 1:13-8.

38. Cainap C, Qin S, Huang WT, et al. Linifanib versus Sorafenib in patients with advanced hepatocellular 
carcinoma: results of a randomized phase III trial. J Clin Oncol 2015;33:172-9.

39. Johnson PJ, Qin S, Park JW, et al. Brivanib versus sorafenib as first-line therapy in patients with unresectable, advanced hepatocellular carcinoma: results from the randomized phase III BRISK-FL study. J Clin Oncol 2013;31:3517-24.

40. Cheng AL, Kang YK, Lin DY, et al. Sunitinib versus sorafenib in advanced hepatocellular cancer: results of a randomized phase III trial. J Clin Oncol 2013;31:4067-75.

41. Ebos JM, Kerbel RS. Antiangiogenic therapy: impact on invasion, disease progression, and metastasis. Nat Rev Clin Oncol 2011;8:210-21.

42. He M, Li Q, Zou R, et al. Sorafenib Plus Hepatic Arterial Infusion of Oxaliplatin, Fluorouracil, and Leucovorin vs Sorafenib Alone for Hepatocellular Carcinoma With Portal Vein Invasion: A Randomized Clinical Trial. JAMA Oncol 2019;5:953-60.

43. Mazzaferro V, Citterio D, Bhoori S, et al. Liver transplantation in hepatocellular carcinoma after tumour downstaging (XXL): a randomised, controlled, phase 2b/3 trial. Lancet Oncol 2020;21:947-56.

44. Sharma P, Allison JP. Immune checkpoint targeting in cancer therapy: toward combination strategies with curative potential. Cell 2015;161:205-14.

45. Dendy MS, Ludwig JM, Stein SM, et al. Locoregional Therapy, Immunotherapy and the Combination in Hepatocellular Carcinoma: Future Directions. Liver Cancer 2019;8:326-40.

46. Kudo M. Combination Cancer Immunotherapy with Molecular Targeted Agents/Anti-CTLA-4 Antibody for Hepatocellular Carcinoma. Liver Cancer 2019;8:1-11.

47. Mizukoshi E, Yamashita T, Arai K, et al. Enhancement of tumor-associated antigen-specific $\mathrm{T}$ cell responses by radiofrequency ablation of hepatocellular carcinoma. Hepatology 2013;57:1448-57.

48. Gu Y-K, editor. Hepatic artery infusion chemotherapy combined with apatinib and toripalimab in advanced hepatocellular carcinoma: Real-world data from a single center2020; ASCO Virtual Scientific Program: American Society of Clinical Oncology.

49. Llovet JM, El-Khoueiry AB, Vogel A, et al. 1016TiP LEAP-012 trial in progress: Pembrolizumab plus lenvatinib and transarterial chemoembolization (TACE) in patients with intermediate-stage hepatocellular carcinoma (HCC) not amenable to curative treatment. Ann Oncol 2020;31:S702-3.

50. Woo HY, Heo J. New perspectives on the management of hepatocellular carcinoma with portal vein thrombosis. Clin Mol Hepatol 2015;21:115-21.

51. Costentin CE, Ferrone CR, Arellano RS, et al.

Hepatocellular Carcinoma with Macrovascular Invasion: Defining the Optimal Treatment Strategy. Liver Cancer 2017;6:360-74.

52. Kudo M. A New Treatment Option for Intermediate-Stage Hepatocellular Carcinoma with High Tumor Burden: Initial Lenvatinib Therapy with Subsequent Selective TACE. Liver Cancer 2019;8:299-311.

53. Kudo M, Ueshima K, Chan S, et al. Lenvatinib as an Initial Treatment in Patients with Intermediate-Stage Hepatocellular Carcinoma Beyond Up-To-Seven Criteria and Child-Pugh A Liver Function: A Proof-Of-Concept Study. Cancers (Basel) 2019;11:1084.

54. Kasai Y, Hatano E, Seo S, et al. Proposal of selection criteria for operative resection of hepatocellular carcinoma with inferior vena cava tumor thrombus incorporating hepatic arterial infusion chemotherapy. Surgery 2017;162:742-51.

55. Lau WY, Lai EC. Salvage surgery following downstaging of unresectable hepatocellular carcinoma--a strategy to increase resectability. Ann Surg Oncol 2007;14:3301-9.

56. Zhou XD, Tang ZY, Ma ZC, et al. Twenty-year survivors after resection for hepatocellular carcinoma-analysis of 53 cases. J Cancer Res Clin Oncol 2009;135:1067-72.

57. Lee MS, Ryoo BY, Hsu CH, et al. Atezolizumab with or without bevacizumab in unresectable hepatocellular carcinoma (GO30140): an open-label, multicentre, phase 1b study. Lancet Oncol 2020;21:808-20.

58. Shigeta K, Datta M, Hato T, et al. Dual Programmed Death Receptor-1 and Vascular Endothelial Growth Factor Receptor-2 Blockade Promotes Vascular Normalization and Enhances Antitumor Immune Responses in Hepatocellular Carcinoma. Hepatology 2020;71:1247-61.

59. Voron T, Marcheteau E, Pernot S, et al. Control of the immune response by pro-angiogenic factors. Front Oncol 2014;4:70.

60. Aoki T, Kudo M, Ueshima K, et al. Exploratory Analysis of Lenvatinib Therapy in Patients with Unresectable Hepatocellular Carcinoma Who Have Failed Prior PD-1/PD-L1 Checkpoint Blockade. Cancers (Basel) 2020;12:3048

61. Osa A, Uenami T, Koyama S, et al. Clinical implications of monitoring nivolumab immunokinetics in non-small cell lung cancer patients. JCI Insight 2018;3:e59125.

62. Harding JJ, Nandakumar S, Armenia J, et al. Prospective 
Genotyping of Hepatocellular Carcinoma: Clinical Implications of Next-Generation Sequencing for Matching Patients to Targeted and Immune Therapies. Clin Cancer Res 2019;25:2116-26.

63. Kudo M. Gd-EOB-DTPA-MRI Could Predict WNT/ $\beta$-Catenin Mutation and Resistance to Immune Checkpoint Inhibitor Therapy in Hepatocellular Carcinoma. Liver Cancer 2020;9:479-90.

Cite this article as: Zhang $\mathrm{T}$, Merle $\mathrm{P}$, Wang $\mathrm{H}$, Zhao $\mathrm{H}$, Kudo M. Combination therapy for advanced hepatocellular carcinoma: do we see the light at the end of the tunnel? HepatoBiliary Surg Nutr 2021;10(2):180-192. doi: 10.21037/ hbsn-2021-7
64. Yamauchi M, Ono A, Ishikawa A, et al. Tumor Fibroblast Growth Factor Receptor 4 Level Predicts the Efficacy of Lenvatinib in Patients With Advanced Hepatocellular Carcinoma. Clin Transl Gastroenterol 2020;11:e0179.

65. Twyman-Saint Victor C, Rech AJ, Maity A, et al. Radiation and dual checkpoint blockade activate nonredundant immune mechanisms in cancer. Nature 2015;520:373-7. 